\title{
Electronically excited states of tryptamine and its microhydrated complex
}

\author{
Michael Schmitt ${ }^{\mathrm{a})}$ and Robert Brause \\ Institut für Physikalische Chemie, Heinrich-Heine-Universität, D-40225 Düsseldorf, Germany \\ Christel M. Marian ${ }^{\text {b) }}$ and Susanne Salzmann \\ Institut für Theoretische Chemie und Computerchemie, Heinrich-Heine-Universität, \\ D-40225 Düsseldorf, Germany \\ W. Leo Meerts ${ }^{\text {c) }}$ \\ Molecular and Biophysics Group, Institute for Molecules and Materials, Radboud University Nijmegen, \\ P.O. Box 9010, NL-6500 GL Nijmegen, The Netherlands
}

(Received 21 June 2006; accepted 21 August 2006; published online 27 September 2006)

\begin{abstract}
The lowest electronically excited singlet states of tryptamine and the tryptamine $\left(\mathrm{H}_{2} \mathrm{O}\right)_{1}$ cluster have been studied, using time dependent density functional theory for determination of the geometries and multireference configuration interaction for the vertical and adiabatic excitation energies, the permanent dipole moments, and the transition dipole moment orientations. All molecular properties of the seven experimentally observed conformers of tryptamine could be reproduced with high accuracy. A strong solvent reorientation has been found upon electronic excitation of the 1:1 water cluster of tryptamine to the $L_{a}$ and $L_{b}$ states. The adiabatically lowest excited singlet state in case of the tryptamine monomer is the $L_{b}$ state, while for the 1:1 water complex, the $L_{a}$ is calculated below the $L_{b}$ state. (C) 2006 American Institute of Physics. [DOI: 10.1063/1.2354494]
\end{abstract}

\section{INTRODUCTION}

The energetic ordering and nature of electronically excited states of 3-substituted indoles is of considerable interest for an understanding of protein fluorescence of tryptophan containing peptides at a molecular level. A multitude of theoretical and gas phase experimental studies have been performed on the indole chromophore itself and on 3-alkyl substituted indoles. ${ }^{1-21}$ The fluorescence properties of tryptophan residues are known to depend strongly on the local surrounding and on solvation. Tryptamine, as important part of the tryptophan residue, is a relatively large system, which is currently close to the limit of high level theoretical descriptions using multireference schemes, and it is also at the edge of the experimental determination of the excited state structure. Therefore, the investigation of properties of individual conformers with the indole chromophore contributes to the understanding of the complex photophysical properties of tryptophan containing peptides. Most of these photophysical properties are explained in the light of two electronically excited singlet states, which are labeled by ${ }^{1} L_{a}$ and ${ }^{1} L_{b}$. The energetic separation of these states depends critically on the electronic nature of the chromophore, i.e., on position and electronic properties of attached substituents, on the local surrounding of the chromophore, and on solvation effects. Since both states differ considerably in their permanent dipole moments, they experience very different stabilizations upon solvation. An investigation of these solvation effects on the splitting and ordering of electronic states has to be per-

\footnotetext{
${ }^{a)}$ Electronic mail: mschmitt@uni-duesseldorf.de

homepage: http://www-public.rz.uni-duesseldorf.de/mschmitt/

b) Electronic mail: christel.marian@uni-duesseldorf.de

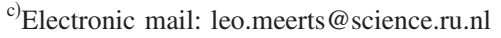

formed with molecules containing all or most of the relevant functional groups, which might act as binding motifs for solvent molecules. In that respect, tryptamine is a good candidate, since it constitutes a model, which still can be interpreted at a detailed molecular level, but in contrast to model systems such as indole or methyl indole possesses the ethyl amino functional group that is responsible for the rich conformer landscape of this molecule and furthermore acts as a binding group for the solvent water molecules.

In a recent publication we presented an analysis of the conformational landscape of the neurotransmitter tryptamine in the electronic ground state using rotationally resolved electronic spectroscopy. ${ }^{19}$ While high quality structure calculations for the electronic ground state are straightforward and bear in general no problems, electronically excited states are much harder to describe with an accuracy comparable to the experiment. Rotationally resolved electronic spectroscopy yields rotational constants which are related to the moments of inertia as primary structural information. To avoid or minimize the risk of accidentally good agreement between experimental and calculated rotational constants, a set of different isotopomers has to be studied. The second motivation for the study of excited states of the tryptamine conformers is the fact that different conformers of one molecule might exhibit very different transition dipole moment orientations upon $S_{1} \leftarrow S_{0}$ excitation. The flexible ethyl amino side chain of tryptamine gives rise, in principle, to 27 conformers from rotations about the two $\mathrm{CC}$ and $\mathrm{CN}$ single bonds. Of these 27 conformers the nine conformers with the $\mathrm{C}_{\alpha} \mathrm{H}_{2}-\mathrm{NH}_{2}$ side chain pointing out of the indole plane were supposed to have considerably lower energies than the remaining 18 ones.

Park et al. found six different conformers of tryptamine in the low resolution laser-induced fluorescence (LIF) spec- 
tra and named them $A$ to $F{ }^{3}$ With higher resolution Philips and Levy ${ }^{4}$ could show that the $C$ band comprises two close $Q$ branches, which were assigned to different conformers (C1 and $C 2$ ). Connell et al. performed an analysis of the conformational landscape using rotational coherence spectroscopy. ${ }^{5}$ The $A$ and $B$ conformers of tryptamine have been observed using microwave spectroscopy. ${ }^{16}$ In this study no further conformers could be found.

Recently, Nguyen et al. ${ }^{18}$ and Schmitt et al. ${ }^{19}$ independently presented the results of high resolution rotationally resolved spectroscopy on the conformers of tryptamine, which differ in the number of conformers which were observed and in two assignments. In the study of Nguyen et al. ${ }^{18}$ seven different conformers were identified, while Schmitt et al. ${ }^{19}$ found only six conformers. The "missing" conformer in the latter study can be identified as the $C 2$ band of Philips and Levy. ${ }^{4}$ In the meantime we were able to spot the $C 2$ band by using a larger nozzle diameter for the molecular beam. This finding clearly points to some degree of kinetic control of the relative amount of the conformers in the molecular beam. ${ }^{22}$ Nguyen and Pratt determined in a recent study the dipole moments of four tryptamine conformers in the electronic ground and excited state using the Stark effect on rotationally resolved electronic spectra. ${ }^{21}$

Carney and Zwier investigated the seven conformers from Ref. 13 in the region of the alkyl C-H stretching vibrations using resonant ion dip infrared (RIDIR) spectroscopy and UV-UV hole-burning spectroscopy. They distinguished and assigned the seven conformers by their different IR spectra. Recently, the energy thresholds between the different conformers were measured by Dian et al. using stimulated emission pumping-hole filling and stimulated emission pumping-induced population transfer spectroscopy ${ }^{17}$ and were compared to the results of a RIMP2/aug-cc-pVDZ study of the respective stationary points on the potential energy surface. ${ }^{20}$

Complexation of tryptamine with one solvent molecule such water, methanol, or ethanol leads to a selective stabilization of only one conformer. ${ }^{23,24}$ The rotational constants of the water cluster were determined by Felker ${ }^{25}$ and Connell et $a l^{26}$ using rotational coherence spectroscopy, and by Schmitt et al. ${ }^{19}$ using rotationally resolved electronic spectroscopy. A bridged structure, which connects the amino group and the acidic $\mathrm{CH}$ group at the pyrrole ring, was found for this cluster, selectively stabilizing the $A[\mathrm{Gpy}$ (out)] conformer. Zwier ${ }^{27}$ performed RIDIR spectroscopy and showed that the bonding to the amino group is considerably stronger than that to the pyrrolic $\mathrm{CH}$ group. Carney et al. investigated higher tryptamine (water) ${ }_{n}$ clusters with $n=2,3$ using RIDIR spectroscopy and could show that water molecules bridge the amino (acceptor) and the indole $\mathrm{NH}$ (donor) group. ${ }^{28}$

In the present study, the lowest excited singlet states of tryptamine and its water cluster are investigated by quantum chemical methods and are compared to the experimental results.

\section{THEORETICAL METHODS AND COMPUTATIONAL DETAILS}

\section{A. Geometry optimization}

Calculations were performed employing the valence triple zeta basis set with polarization functions $(d, p)$ from the TURBOMOLE library. ${ }^{29,30}$ The equilibrium geometry of the electronic ground state was determined for a restricted closed shell Kohn-Sham (KS) determinant using the B3-LYP density functional. ${ }^{31,32}$ Furthermore, we optimized the geometries of the low-lying $L_{a}$ and $L_{b}$ electronic singlet states by means of a time-dependent density functional theory (TDDFT) gradient. ${ }^{33}$ All DFT or TDDFT calculations were carried out utilizing the TURBOMOLE package, version 5.6. ${ }^{34}$

Second-order perturbation theory (MP2) optimizations were performed with the 6-311 $\mathrm{G}(d, p)$ basis set using the GAUSSIAN 03 program package (Revision A.1). ${ }^{35}$ The selfconsistent field (SCF) convergence criterion used throughout the calculations was an energy change below $10^{-8}$ hartree, and the convergence criterion for the gradient optimization of the molecular geometry was $\partial E / \partial r<1.5$ $\times 10^{-5}$ hartree/bohr and $\partial E / \partial \varphi<1.5 \times 10^{-5}$ hartree/deg, respectively. The ZPE corrections were performed with the vibrational frequencies computed using the analytical second derivatives.

Single point MP2 calculations within the resolution-ofthe-identity (RI) approximation were accomplished with the TURBOMOLE program package (Version 5.8$)^{36-38}$ yielding the MP2 stabilization energies at the MP2/6-311G $(d, p)$ optimized structures. As appropriate basis sets Dunning's (augmented) correlation consistent valence quadruple $\zeta$ with polarization functions was used, enhancing the number of basis functions to 1020 (1512). ${ }^{39}$ The complete basis set model CBS-4 of Ochterski et al. (Ref. 40) has been used with the new localization procedure and improved empirical parameters from Ref. 41 as implemented in the GAUSSIAN 03 program package.

\section{B. Electronic excitation energies}

For the computation of singlet and triplet state energies and wave functions, we used the combined density functional theory/multireference configuration interaction (DFT/ MRCI) method by Grimme and Waletzke. ${ }^{42}$ This method was shown to yield excellent electronic spectra of organic molecules at reasonable computational expense. The configuration state functions (CSFs) in the MRCI expansion are built up from KS orbitals, optimized for the dominant closed shell determinant of the electronic ground state employing the BH-LYP (Refs. 32 and 43) functional. All 62 valence electrons were correlated in the MRCI runs, and the eigenvalues and eigenvectors of eight singlet states were determined. The initial set of reference CSFs was generated automatically in a complete-active-space-type procedure (including all single excitations from the five highest occupied molecular orbitals in the KS determinant to the five lowest virtual orbitals) and was then iteratively improved. The MRCI expansion was kept moderate by extensive configuration selection. The MRCI space was spanned by about 
TABLE I. Relative stabilities $\Delta E\left(\mathrm{~cm}^{-1}\right)$ of the seven most stable tryptamine conformers. All calculations include ZPE corrections at the level of the optimization. The method in the first line of each column gives the level for the energy calculation, and the method in the second line the level of optimization and ZPE correction.

\begin{tabular}{lccccr}
\hline \hline & & \multicolumn{4}{c}{ RIMP2 } \\
\cline { 3 - 5 } Energy \\
\cline { 3 - 5 } optimization & $\begin{array}{c}\text { DFT/MRCI } \\
\text { TZVP } \\
\text { DFT/TZVP }\end{array}$ & $\begin{array}{c}\text { MP2/6-311G }(d, p) \\
\text { MPc-pVQZ }\end{array}$ & $\begin{array}{c}\text { aug-cc-pVQZ } \\
\text { MP2/6-311G }(d, p)\end{array}$ & MP2/6-311G $(d, p)$ & CBS-4M \\
\hline Gpy(out) & 0 & 0 & 0 & 0 & 0 \\
Gpy(up) & 244 & 21 & 148 & 177 & 144 \\
Gph(out) & 20 & 1 & 201 & 243 & 276 \\
Gph(up) & 157 & 128 & 286 & 337 & 378 \\
Anti(py) & 371 & 509 & 486 & 497 & 457 \\
Anti(ph) & 229 & 520 & 486 & 497 & 447 \\
Anti(up) & 341 & 444 & 503 & 538 & 475 \\
\hline \hline
\end{tabular}

100000 energy-selected single and double excitations (200000 singlet CSFs) from approximately 50 reference configurations.

\section{RESULTS AND DISCUSSION}

\section{A. Electronic ground state energies and structures}

The electronic ground state energies of the seven experimentally observed conformers of tryptamine are calculated using DFT/MRCI and are compared in Table I to the results of calculations using other methods. The structures of the conformers under investigation in this study are shown in Fig. 1. The nomenclature for the orientation of the ethyl amino side chain in the different conformers which was introduced by Carney et al. is used here. ${ }^{13}$ Gpy (Gph) designates conformations in which the amino group is in gauche conformation and points to the pyrrole (phenyl) side of the chromophore. In all anti conformers, the amino group points away from the chromophore. The orientation of the amino group lone pair is described by the identifiers "up," "out," "in," "py," and "ph." Although the two in conformers, with the lone pair pointing to the indole ring, have similar ground state energies as the other seven ones, they have never been observed experimentally.

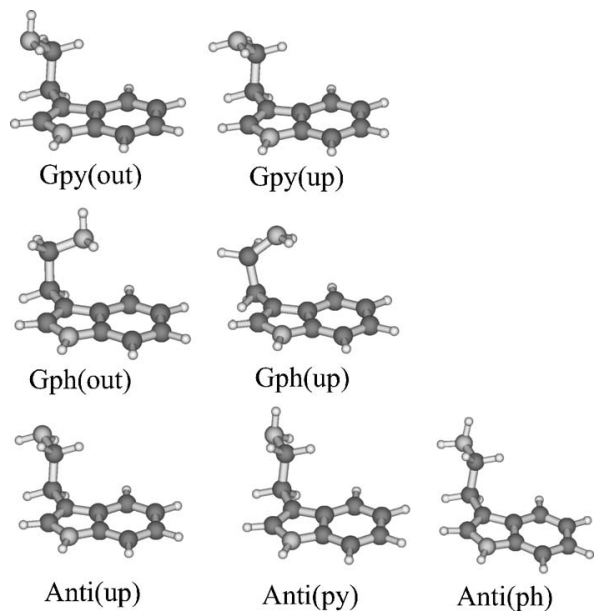

FIG. 1. Ground state structures of the seven experimentally observed tryptamine conformers.
As a general trend for the ground state energies of the DFT/MRCI as well as the MP2 calculations, it is found that the ground state energies of the three anti conformers (py, ph, and up) are considerably higher than those of the out and up forms of Gpy and Gph. The most stable conformer with all methods is the Gpy(out) form. This finding can be explained by an additional stabilization of the Gpy and Gph forms through the dispersion interaction between the amino group and the aromatic system, which cannot be formed in the anti conformers. Clarkson et al. could show that $\operatorname{CCSD}(\mathrm{T})$ calculations at the B3LYP optimized structure are able to reproduce the trend of the MP2 ground state energies, while successively larger basis sets used with the DFT method did not improve the results. ${ }^{20}$ Therefore, they concluded that basis set superposition errors (BSSEs) from the interaction of the amino group orbitals with the indole ring are not the major source of the discrepancies. We have calculated the relative stabilization energies of the seven conformers at the respective MP2/6-311G $(d, p)$ optimized geometries with RIMP2 and successively increasing basis sets (Table I). All energies have been zero-point energy (ZPE) corrected using the vibrational frequencies at the level of optimization. Extrapolating the data from Table I to the basis set limit shows that the anti(up) conformer is the most unstable one, followed by the two other anti conformers. Using the largest basis sets, both Gpy conformers are more stable than the Gph conformers.

The rotational constants from the optimized DFT calculations are in good agreement with the experimental values (cf. Tables S1 and S2 of the supplemental material ${ }^{44}$ ), although the quality of the best MP2 calculations is not fully reached. Clearly, the discrepancy can be traced back to the underevaluation of dispersion interactions, in the DFT method. Proper consideration of electron correlation as in MP2 tends to stabilize the gauche conformers via dispersive interactions between the ethyl amino group and the aromatic ring. This is immediately obvious from the root mean square deviation (RMSD) of the experimental and calculated rotational constants. The RMSD for the rotational constants of all conformers of the MP2 calculations amounts to $7.5 \mathrm{MHz}$, while for the B3LYP calculations it is considerably larger $(12.6 \mathrm{MHz})$. This effect is mainly due to the neglect of dispersion interaction in the DFT calculations, since the RMSD 
TABLE II. Calculated vertical and adiabatic singlet excitation energies $\Delta E\left(\mathrm{~cm}^{-1}\right)$ of seven tryptamine conformers and the tryptamine [Gpy(out)]-water cluster. Adiabatic excitation energies are ZPE corrected by the amount given in parentheses. The adiabatic excitation energies of the water cluster contain BSSE corrections at the DFT/MRCI level. Electric dipole transition oscillator strengths $f(r)$ are displayed in the second row for each conformer in parentheses.

\begin{tabular}{|c|c|c|c|c|c|c|c|}
\hline \multirow[b]{2}{*}{ Geometry state } & \multicolumn{2}{|c|}{$\mathrm{S}_{0} \min$} & \multicolumn{2}{|c|}{$L_{b} \min$} & \multicolumn{2}{|c|}{$L_{a} \min$} & \multirow[b]{2}{*}{ Expt. } \\
\hline & $L_{b}$ & $L_{a}$ & $L_{b}$ & $L_{a}$ & $L_{b}$ & $L_{a}$ & \\
\hline Gpy(out) & $\begin{array}{l}36900 \\
(0.022)\end{array}$ & $\begin{array}{l}39170 \\
(0.136)\end{array}$ & $\begin{array}{c}34841(1059) \\
(0.027)\end{array}$ & $\begin{array}{l}39010 \\
(0.142)\end{array}$ & $\begin{array}{l}38990 \\
(0.011)\end{array}$ & $\begin{array}{c}35726(1174) \\
(0.120)\end{array}$ & 34916 \\
\hline Gpy(up) & $\begin{array}{l}36730 \\
(0.024)\end{array}$ & $\begin{array}{l}39120 \\
(0.131)\end{array}$ & $\begin{array}{c}34636(1024) \\
(0.028)\end{array}$ & $\begin{array}{l}38780 \\
(0.147)\end{array}$ & $\begin{array}{l}38880 \\
(0.009)\end{array}$ & $\begin{array}{c}35949(1091) \\
(0.124)\end{array}$ & 34896 \\
\hline Gph(out) & $\begin{array}{l}36850 \\
(0.025)\end{array}$ & $\begin{array}{l}39402 \\
(0.151)\end{array}$ & $\begin{array}{c}34885(1045) \\
(0.025)\end{array}$ & $\begin{array}{l}39100 \\
(0.152)\end{array}$ & $\begin{array}{l}39010 \\
(0.012)\end{array}$ & $\begin{array}{c}35946(1074) \\
(0.127)\end{array}$ & 34879 \\
\hline Gph(up) & $\begin{array}{l}36780 \\
(0.022)\end{array}$ & $\begin{array}{l}38860 \\
(0.148)\end{array}$ & $\begin{array}{c}34608(1162) \\
(0.026)\end{array}$ & $\begin{array}{l}38730 \\
(0.149)\end{array}$ & $\begin{array}{l}37690 \\
(0.009)\end{array}$ & $\begin{array}{c}35886(1004) \\
(0.219)\end{array}$ & 34832 \\
\hline Anti(up) & $\begin{array}{l}36560 \\
(0.018)\end{array}$ & $\begin{array}{l}38810 \\
(0.131)\end{array}$ & $\begin{array}{c}34568(1072) \\
(0.025)\end{array}$ & $\begin{array}{l}38670 \\
(0.142)\end{array}$ & $\begin{array}{l}38410 \\
(0.009)\end{array}$ & $\begin{array}{c}35 \text { 603(887) } \\
(0.123)\end{array}$ & 34868 \\
\hline Anti(py) & $\begin{array}{l}36670 \\
(0.019)\end{array}$ & $\begin{array}{l}38920 \\
(0.132)\end{array}$ & $\begin{array}{c}34628(1032) \\
(0.028)\end{array}$ & $\begin{array}{l}38770 \\
(0.147)\end{array}$ & $\begin{array}{l}38630 \\
(0.011)\end{array}$ & $\begin{array}{c}35759(891) \\
(0.122)\end{array}$ & 34884 \\
\hline Anti(ph) & $\begin{array}{l}36710 \\
(0.021)\end{array}$ & $\begin{array}{l}39160 \\
(0.135)\end{array}$ & $\begin{array}{c}34 \text { 696(984) } \\
(0.029)\end{array}$ & $\begin{array}{l}38910 \\
(0.148)\end{array}$ & $\begin{array}{l}38660 \\
(0.011)\end{array}$ & $\begin{array}{c}36508(372) \\
(0.125)\end{array}$ & 34880 \\
\hline $\mathrm{Gpy}($ out $)-\mathrm{H}_{2} \mathrm{O}$ & $\begin{array}{l}36845 \\
(0.019)\end{array}$ & $\begin{array}{l}38947 \\
(0.133)\end{array}$ & $\begin{array}{c}34872(837) \\
(0.021)\end{array}$ & $\begin{array}{l}37449 \\
(0.119)\end{array}$ & $\begin{array}{l}35817 \\
(0.011)\end{array}$ & $\begin{array}{c}33 \text { 931(906) } \\
(0.113)\end{array}$ & 34957 \\
\hline
\end{tabular}

of the anti conformers alone is nearly equal for MP2 and B3LYP (7 and $9.5 \mathrm{MHz}$, respectively), while it differs considerably for the $\mathrm{Gph} / \mathrm{Gpy}$ conformers $(7.8$ and $14.9 \mathrm{MHz}$, respectively).

\section{B. Calculated vertical and adiabatic absorption spectra}

\section{Vertical absorption spectra}

Vertical and adiabatic excitation energies of the $L_{b}$ and $L_{a}$ states of the seven lowest energy conformers are shown in Table II along with the respective electric dipole oscillator strengths. At the respective ground state equilibrium geometries, the first ${ }^{1}\left(\pi \rightarrow \pi^{*}\right) L_{b}$ states of all conformers exhibit significant configuration mixing. The leading configuration corresponds to an excitation from the second highest occupied molecular orbital (HOMO-1) to the lowest unoccupied molecular orbital (LUMO). In addition, the excitation from the HOMO to the LUMO+ 1 has considerable weight, but its coefficient in the configuration interaction (CI) expansion has a different phase. Due to cancellation effects of the individual contributions of the two configurations to the electric transition dipole moment, the electric dipole transition to this state, commonly denominated as $L_{b}$, is weak. The optically bright $L_{a}$ state $(\mathrm{HOMO} \rightarrow \mathrm{LUMO})$ constitutes only the second excited singlet state in the vertical absorption spectrum, about 2000-2500 $\mathrm{cm}^{-1}$ above the $L_{b}$ state, depending on the conformer.

For the adiabatic excitations the ZPE corrections have been calculated, using the analytical second derivatives for the ground state and numerical second derivatives for the $L_{a}$ and $L_{b}$ states. For all conformers negative corrections for the excitation energies $\left[\mathrm{ZPE}\left(L_{a} / L_{b}\right)-\mathrm{ZPE}\left(S_{0}\right)\right]$ have been found, showing an overall decrease of the sum of the vibrational frequencies upon electronic excitation. While for excitation to the $L_{b}$ state the corrections are nearly constant $\left(-1000 \mathrm{~cm}^{-1}\right)$ for all conformers, the ZPE differences vary strongly with the conformer for excitation to the $L_{a}$ state. Especially, the anti conformers have smaller corrections than the Gph and Gpy conformers. The smallest ZPE correction is found for excitation to the $L_{a}$ state of anti( $\left.\mathrm{ph}\right)$. Inspection of the respective vibrational frequencies shows that this effect can be traced back to lower vibrational frequencies in the $L_{a}$ state of this conformer compared to all other ones. Thus, it is not an effect of selected modes, compensating each other but arising from an overall similarity of ground and excited state vibrational frequencies for that conformer. Molden frequency 


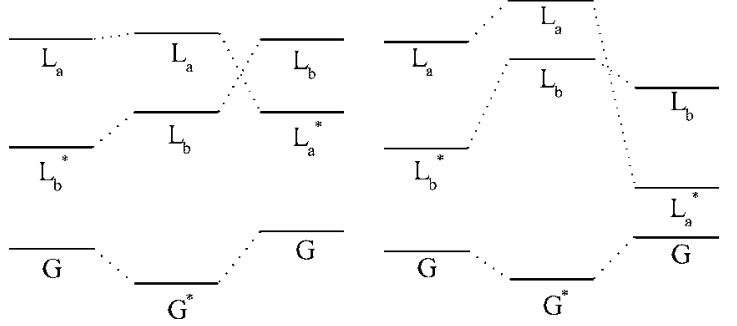

FIG. 2. Schematic geometry relaxations of tryptamine (left diagram) and tryptamine-water (right diagram) upon electronic excitation. The respective fully optimized state is marked with an asterisk. The other states given in the same column are calculated in the geometry of the optimized state.

files (.molf) and Gaussian (.log) files containing geometries and vibrational modes of all tryptamine conformers investigated in this study can be obtained from the authors' home page.

\section{2. $L_{a}$ minimum structures}

Starting the TDDFT $S_{1}$ minimum search of a conformer from its $S_{0}$ minimum nuclear arrangement leads the gradient toward a local minimum on the $S_{1}$ potential energy hypersurface. Its electronic structure corresponds to the $L_{a}$ state. Given that the $L_{b}$ state is the lowest excited singlet state at the ground state equilibrium geometry (see Fig. 2), we had expected to find the $L_{b}$ minimum instead. It turned out, however, that TDDFT employing the B3-LYP functional reverses the order of the $L_{a}$ and $L_{b}$ states with respect to the DFT/ MRCI method. As will be shown in Sec. III D, experimental evidence clearly shows that the ordering of states obtained by the DFT/MRCI method is the correct one. At DFT/MRCI level of treatment, the $L_{b}$ state is the second excited singlet state at the $L_{a}$ minimum, approximately $2000 \mathrm{~cm}^{-1}$ above the $L_{a}$.

\section{3. $L_{b}$ minimum structures}

The optimization of the $L_{b}$ state of all conformers is complicated by the fact that it constitutes only the second excited singlet state at the TDDFT level. Using the DFT/ MRCI method at the TDDFT optimized geometry, this state is located adiabatically $1000-1500 \mathrm{~cm}^{-1}$ below the $L_{a}$ state for all conformers and constitutes the $S_{1}$ state, while the $L_{a}$ is the second state in the singlet manifold. This means that the electronic structure of the $S_{1}$ state swaps about halfway along the reaction coordinate connecting the $S_{0}$ and $S_{1}\left(L_{a}\right)$ minimum geometries. We therefore predict strong vibronic interaction between $L_{a}$ and $L_{b}$ states.

Figure 2 summarizes schematically the trends for the vertical and adiabatic excitation energies of an arbitrary conformer of tryptamine. The asterisk denotes the states which have been optimized. Thus, from left to right the adiabatic excitation to the $L_{b}$ state, the vertical excitation to $L_{a}$ and $L_{b}$ states and the adiabatic excitation to the $L_{a}$ state are depicted. Clearly, the ${ }^{1} L_{b}$ state constitutes the lowest excited state, both in the vertical and in the adiabatic excitation spectrum. Figure 3 compares the adiabatic excitation energies of the $L_{a}$ and $L_{b}$ states of the seven experimentally observed tryptamine conformers including the respective ZPE correc-

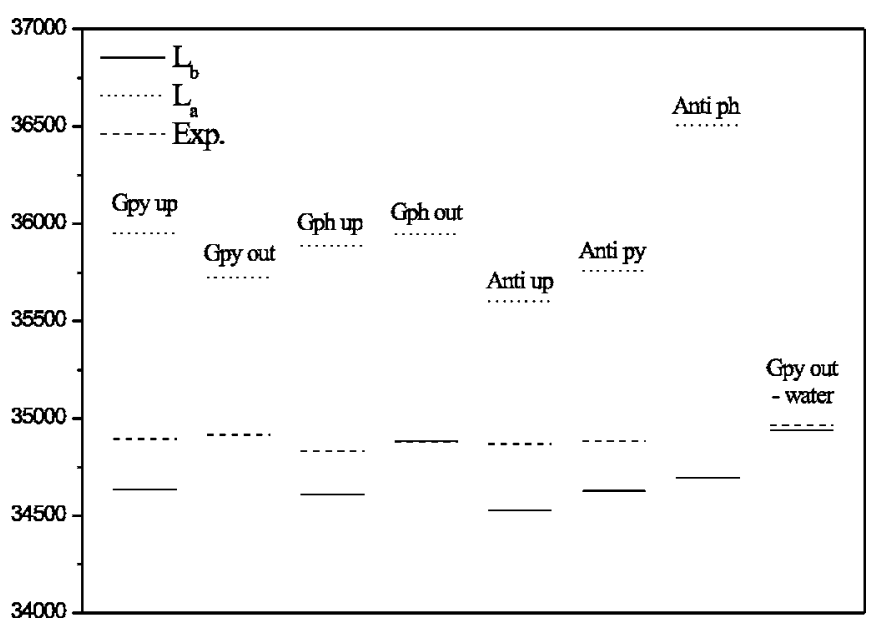

FIG. 3. Adiabatic excitation energies of the $L_{a}$ (dotted lines) and $L_{b}$ (straight lines) states of the seven experimentally observed tryptamine conformers and the tryptamine-water cluster. Dashed lines represent the experimental excitation energies of the lowest energy transition.

tions. The numerical agreement between the $L_{b}$ excitation energies and the experimentally determined origins is striking. Since the electronic origins of all seven conformers are located within $84 \mathrm{~cm}^{-1}$, the accuracy of the method would be overstrained if one expects the ordering of the excitation energies of all conformers to be reproduced. Nevertheless, even some of the features are reproduced satisfactorily. Both Gph and Gpy conformer classes have the excitation energies of the out form at higher energy compared to the up form and the excitation energies within the anti class increases in the orders up, py, and ph.

\section{Structural changes upon electronic excitation}

The calculated principal distortions of the geometry of tryptamine upon electronic excitation to the $L_{a}$ and the $L_{b}$ states do not depend strongly on the conformer. Figure 4

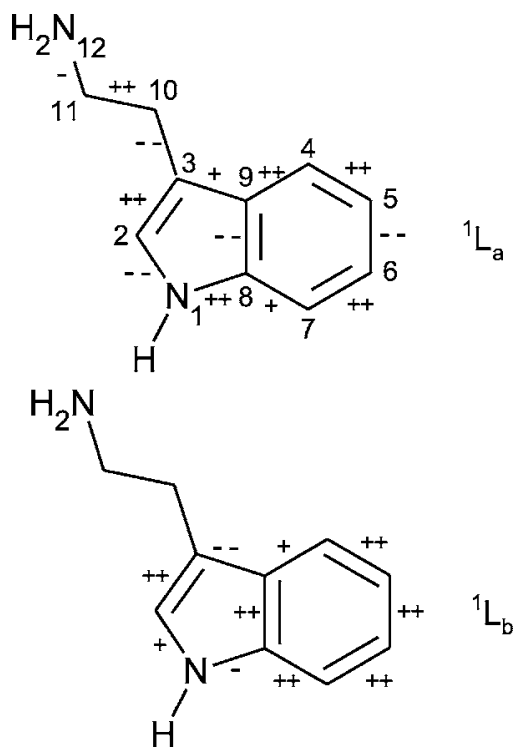

FIG. 4. Schematic structural changes upon electronic excitation to the $L_{a}$ and $L_{b}$ states of tryptamine. $+(-)$ indicates a bond length increase (decrease) between 0.2 and $1.5 \mathrm{pm}$, and $++(--)$ a change of more than $1.5 \mathrm{pm}$. A missing indicator designates changes between -0.2 and $+0.2 \mathrm{pm}$. 
shows the approximate changes of the bond lengths upon electronic excitation. A plus (minus) sign indicates a bond length increase (decrease) between 0.2 and $1.5 \mathrm{pm}$, and a double plus (double minus) sign a change of more than $1.5 \mathrm{pm}$. A missing indicator designates changes between -0.2 and $+0.2 \mathrm{pm}$. The bond length alterations in the $L_{b}$ state are dominated by a more or less symmetric expansion of the benzene ring and alternate bond length changes in the pyrrole ring. The situation changes completely upon excitation to the $L_{a}$ state. Both rings show an unsymmetric overall expansion. The most prominent difference is found in the geometry changes of the ethyl amino side chain. While for the $L_{b}$ state virtually no changes in the $\mathrm{C}-\mathrm{C}$ and $\mathrm{C}-\mathrm{N}$ bond lengths are found, the $L_{a}$ state shows strong alternate bond length changes and very large changes of the dihedral angles, defining the orientation of the side chain.

The different structural changes upon $L_{a}$ and $L_{b}$ excitation can be rationalized with the aid of the frontier orbitals, calculated with DFT/MRCI and shown in the left half of Fig. 5. For the $L_{a}$ excitation which has predominantly HOMO $\rightarrow$ LUMO character, the bond length variations in the chromophore follow nicely the changes in the orbital overlap. Also, the large variations of the bond lengths in the ethyl amino chain can be rationalized from the orbitals in Fig. 5.

For the $L_{b}$ excitation mainly two contributions have to be considered. One is the $\mathrm{HOMO} \rightarrow \mathrm{LUMO}+1$ excitation, the other HOMO-1 $\rightarrow$ LUMO. For all CC bonds in the benzene moiety of the chromophore, excitation occurs from nonbonding or bonding to antibonding orbitals, explaining its overall expansion. At least, for the HOMO- $1 \rightarrow$ LUMO the changes in the ethyl amino chain are small, thus rationalizing the small bond length changes in the side chain.

Geometry changes in tryptamine upon excitation to the $L_{a}$ state are quite different from those of indole, while the general trends for excitation to the $L_{b}$ state are similar for indole and tryptamine. Table III compares the calculated geometry changes upon excitation to the $L_{a}$ and $L_{b}$ states of the chromophore indole, ${ }^{45}$ the Gpy(out) conformer of tryptamine, and the tryptamine-water cluster. Since all tryptamine conformers show very similar geometry changes in the chromophore moiety, we chose the most stable one for the comparison to indole. The geometry parameters of all other conformers are available as online supplementary material or directly from the home page of the authors. The ground state geometry parameters of the chromophore obtained from the B3-LYP/TZVP optimized structure are close to those of indole ${ }^{45}$ which were obtained from optimizations at the complete active space self-consistent field/atomic natural orbital basis sets (CASSCF/ANO-S) level of theory. A common motif of the geometry changes is a moderate overall expansion of the benzene ring and strongly alternating bond length changes in the pyrrole moiety upon $L_{b}$ excitation. The excitation to the $L_{a}$ state leads to considerably diverse structural changes. The only common trends are larger bond length changes in the pyrrole moiety compared to $L_{b}$ excitation. Changes of the angles in the chromophores and in the side chain are generally small in indole and tryptamine. The different behavior upon $L_{a}$ excitation again can be understood comparing the frontier orbitals of tryptamine and in-

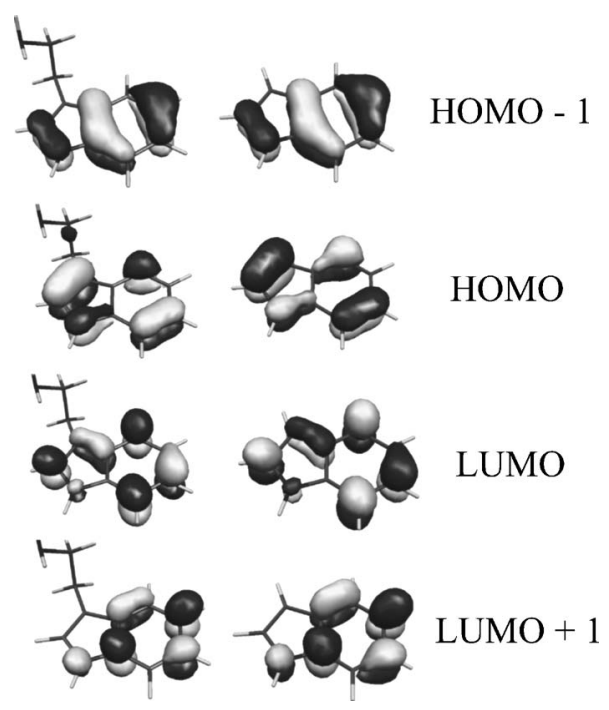

FIG. 5. Kohn-Sham (BH-LYP) calculated frontier orbitals of tryptamine (left) and indole (right) as used in DFT/MRCI calculations. Shown are the orbitals of the Gph(out) conformer; all other conformers are very similar.

dole. Since the $L_{a}$ excitation has predominantly HOMO $\rightarrow$ LUMO character, the strong change of the HOMO (Fig. 5) influences the geometry changes upon excitation to this state much more than to the mixed $(\mathrm{HOMO} \rightarrow$ LUMO $+1 /$ HOMO $-1 \rightarrow$ LUMO) $L_{b}$ state.

\section{Permanent dipole moments and transition dipoles}

Recently, Nguyen and Pratt determined the permanent dipole moments of four tryptamine conformers in the ground and electronically excited state from a rotationally resolved Stark measurement. ${ }^{21}$ These dipole moments can be used to distinguish between the different conformers, since they depend crucially on the position of the ethyl amino chain relative to the chromophore. The experimental dipole moments of the conformers in their electronic ground states are given in Tables S1 and S2 of the online supporting material. The excited state dipole moments are collected in Tables IV and $\mathrm{V}$ and are compared to the results of the DFT/MRCI calculations for the $L_{a}$ and $L_{b}$ states. The agreement for the excited state dipole moment of the $L_{b}$ state is good for the four conformers for which experimental values are available. Although the $L_{a}$ dipole moments are always larger than the respective dipole moments of the $L_{b}$ state, there are large differences between the conformers.

The direction of the transition dipole moment (TDM) is an important indicator of the electronic nature of the excited state. Its orientations with respect to the inertial axes of the respective conformer are defined as

$$
\begin{aligned}
& \mu_{a}=\mu \sin \phi \cos \theta, \\
& \mu_{b}=\mu \sin \phi \sin \theta, \\
& \mu_{c}=\mu \cos \phi,
\end{aligned}
$$

with $\phi$ and $\theta$ as spherical coordinate angles of the transition moment vector in the molecule fixed frame $(a, b, c)$. Tables IV and $\mathrm{V}$ give the experimental and calculated transition 
TABLE III. Ground state geometries and geometry changes of tryptamine [Gpy(out)], tryptamine-water, and indole upon excitation to the $L_{a}$ and the $L_{b}$ states. Distances $r$ and their changes are given in picometers, angles $a$ and dihedral angles $d$ in degrees. For the numbering scheme refer to Figs. 4 and 6.

\begin{tabular}{|c|c|c|c|c|c|c|c|c|c|}
\hline & \multicolumn{3}{|c|}{ Tryptamine } & \multicolumn{3}{|c|}{ Tryptamine-water } & \multicolumn{3}{|c|}{ Indole (Ref. 45) } \\
\hline & $S_{0}$ & $\Delta L_{b}$ & $\Delta L_{a}$ & $S_{0}$ & $\Delta L_{b}$ & $\Delta L_{a}$ & $S_{0}$ & $\Delta L_{b}$ & $\Delta L_{a}$ \\
\hline$r\left(\mathrm{~N}_{1}-\mathrm{C}_{2}\right)$ & 138.3 & 1.2 & -4.4 & 138.1 & 1.7 & -4.6 & 137.9 & 1.4 & -0.3 \\
\hline$r\left(\mathrm{C}_{2}-\mathrm{C}_{3}\right)$ & 136.9 & 2.4 & 6.1 & 137.1 & 2.0 & 6.1 & 136.9 & 1.5 & 10.3 \\
\hline$r\left(\mathrm{C}_{3}-\mathrm{C}_{9}\right)$ & 144.3 & -2.0 & 0.5 & 144.2 & -1.8 & 0.7 & 144.5 & -1.8 & -3.4 \\
\hline$r\left(\mathrm{C}_{4}-\mathrm{C}_{5}\right)$ & 138.5 & 3.5 & 4.5 & 138.6 & 3.4 & 4.5 & 138.8 & 5.8 & -1.4 \\
\hline$r\left(\mathrm{C}_{5}-\mathrm{C}_{6}\right)$ & 140.6 & 2.5 & -2.7 & 140.6 & 2.5 & -2.6 & 141.7 & 2.4 & 1.3 \\
\hline$r\left(\mathrm{C}_{6}-\mathrm{C}_{7}\right)$ & 138.6 & 2.0 & 5.1 & 138.6 & 2.0 & 4.8 & 138.9 & 4.5 & 7.6 \\
\hline$r\left(\mathrm{C}_{7}-\mathrm{C}_{8}\right)$ & 139.5 & 1.7 & 1.4 & 139.5 & 1.8 & 1.7 & 140.5 & 0.7 & -1.9 \\
\hline$r\left(\mathrm{C}_{8}-\mathrm{C}_{9}\right)$ & 142.0 & 4.0 & -3.2 & 142.1 & 3.9 & -3.5 & 140.8 & 5.5 & 2.4 \\
\hline$r\left(\mathrm{~N}_{1}-\mathrm{C}_{8}\right)$ & 137.8 & -0.4 & 4.2 & 137.8 & -0.7 & 4.7 & 137.3 & -0.5 & 1.8 \\
\hline$r\left(\mathrm{C}_{4}-\mathrm{C}_{9}\right)$ & 140.3 & 0.8 & 2.0 & 140.3 & 0.8 & 2.0 & 141.0 & 0.9 & 6.1 \\
\hline$r\left(\mathrm{C}_{3}-\mathrm{C}_{10}\right)$ & 150.0 & -0.3 & -2.4 & 150.0 & -0.3 & -2.1 & $\cdots$ & $\cdots$ & $\cdots$ \\
\hline$r\left(\mathrm{C}_{10}-\mathrm{C}_{11}\right)$ & 153.7 & 0.1 & 1.9 & 153.7 & 0.0 & 1.2 & $\cdots$ & $\cdots$ & $\cdots$ \\
\hline$r\left(\mathrm{C}_{11}-\mathrm{N}_{12}\right)$ & 146.5 & 0.0 & -0.7 & 147.4 & 0.0 & 0.0 & $\cdots$ & $\cdots$ & $\cdots$ \\
\hline$r\left(\mathrm{~N}_{12}-\mathrm{H}_{b}\right)$ & $\cdots$ & $\cdots$ & $\cdots$ & 192.9 & 0.1 & -0.7 & $\cdots$ & $\cdots$ & $\cdots$ \\
\hline$r\left(\mathrm{O}_{13}-\mathrm{H}_{2}\right)$ & $\cdots$ & $\cdots$ & $\cdots$ & 252.3 & -8.0 & -17.0 & $\cdots$ & $\cdots$ & $\cdots$ \\
\hline$a\left(\mathrm{~N}_{1} \mathrm{C}_{2} \mathrm{C}_{3}\right)$ & 110.2 & -1.1 & -3.1 & 109.9 & -1.2 & -3.0 & 109.6 & -0.9 & -4.2 \\
\hline$a\left(\mathrm{C}_{2} \mathrm{C}_{3} \mathrm{C}_{9}\right)$ & 106.2 & 1.1 & 1.1 & 106.5 & 1.1 & 1.0 & 106.7 & 1 & 1.1 \\
\hline$a\left(\mathrm{C}_{4} \mathrm{C}_{5} \mathrm{C}_{6}\right)$ & 121.0 & 1.0 & 1.2 & 121.1 & 1.0 & 1.0 & 120.9 & 0.8 & 1.4 \\
\hline$a\left(\mathrm{C}_{5} \mathrm{C}_{6} \mathrm{C}_{7}\right)$ & 121.2 & 0.4 & 1.4 & 121.1 & 0.2 & 1.8 & 121.2 & -0.3 & 1 \\
\hline$a\left(\mathrm{C}_{6} \mathrm{C}_{7} \mathrm{C}_{8}\right)$ & 117.6 & -1.3 & -3.7 & 117.6 & -1.2 & -3.8 & 117.5 & -0.7 & -2.5 \\
\hline$a\left(\mathrm{C}_{2} \mathrm{~N}_{1} \mathrm{C}_{8}\right)$ & 109.1 & 0.9 & 2.5 & 109.4 & 1.2 & 2.3 & 109.3 & 1.3 & 2.9 \\
\hline$a\left(\mathrm{C}_{5} \mathrm{C}_{4} \mathrm{C}_{9}\right)$ & 119.2 & -1.4 & -3.4 & 119.2 & -1.4 & -3.5 & 118.9 & -1.1 & -2.4 \\
\hline$a\left(\mathrm{C}_{4} \mathrm{C}_{9} \mathrm{C}_{3}\right)$ & 134.0 & -0.1 & -1.8 & 134.1 & 0.1 & -2.0 & 133.9 & 0 & -2.2 \\
\hline$a\left(\mathrm{C}_{2} \mathrm{C}_{3} \mathrm{C}_{10}\right)$ & 126.6 & -0.9 & -1.1 & 125.9 & -1.4 & -1.1 & $\cdots$ & $\cdots$ & $\cdots$ \\
\hline$a\left(\mathrm{C}_{3} \mathrm{C}_{10} \mathrm{C}_{11}\right)$ & 114.0 & -0.3 & -1.1 & 113.9 & -0.2 & -0.9 & $\cdots$ & $\cdots$ & $\cdots$ \\
\hline$a\left(\mathrm{C}_{10} \mathrm{C}_{11} \mathrm{~N}_{12}\right)$ & 110.7 & 0.2 & 0.3 & 110.8 & 0.3 & 0.6 & $\cdots$ & $\cdots$ & $\cdots$ \\
\hline$a\left(\mathrm{~N}_{12} \mathrm{H}_{b} \mathrm{O}_{13}\right)$ & $\cdots$ & $\cdots$ & $\cdots$ & 166.4 & -0.8 & -2.7 & $\cdots$ & $\cdots$ & $\cdots$ \\
\hline$d\left(\mathrm{C}_{2} \mathrm{C}_{3} \mathrm{C}_{10} \mathrm{C}_{11}\right)$ & 102.5 & 1.2 & 0.1 & 99.0 & -2.0 & 2.8 & $\cdots$ & $\cdots$ & $\cdots$ \\
\hline$d\left(\mathrm{C}_{3} \mathrm{C}_{10} \mathrm{C}_{11} \mathrm{~N}_{12}\right)$ & -63.9 & -0.2 & 3.0 & -59.6 & -0.4 & 2.8 & $\cdots$ & $\cdots$ & $\cdots$ \\
\hline$d\left(\mathrm{C}_{10} \mathrm{C}_{11} \mathrm{~N}_{12} \mathrm{H}_{12 a}\right)$ & 64.8 & 0.3 & 10.0 & 67.0 & 1.7 & 8.8 & $\cdots$ & $\cdots$ & $\cdots$ \\
\hline
\end{tabular}

dipole moment orientations for all seven conformers. In general, the $L_{a}$ states show transition dipole moments with small angles $\theta$ (close to zero) and large angles $\phi$ (close to $90^{\circ}$ ), while the $L_{b}$ states are characterized by values of $\phi$ and $\theta$ close to $90^{\circ}$. Using the definitions of Eq. (1), one can see that the $L_{a}$ states give rise to predominantly $b$-type bands, while the $L_{b}$ states have the characteristics of $a$-type bands.
The electronic origins of all conformers are predominantly $a$-type bands (cf. Tables IV and V) and can therefore safely be classified as the origins of the $L_{b}$ state. Since both $L_{a}$ and $L_{b}$ states have their respective transition dipole moment nearly parallel to the $b$ and $a$ inertial axes the indeterminacy of the sign of the TDM angle, which arises from the dependence of the intensities from the squares of the TDM components in Eq. (1), is insignificant here.

TABLE IV. Molecular parameters of the excited states of the Gph and Gpy conformers of tryptamine. The calculated transition dipole moment angles $\phi$ and $\theta$ and the permanent dipole moments are calculated by DFT/MRCI in the TDDFT optimized geometry of the respective state. Rotational constants are given in megahertz, dipole moments in debye, and angles of the transition dipole moment in degrees.

\begin{tabular}{|c|c|c|c|c|c|c|c|c|c|c|c|c|}
\hline & \multicolumn{3}{|c|}{ Gpy(out) } & \multicolumn{3}{|c|}{ Gpy(up) } & \multicolumn{3}{|c|}{ Gph(out) } & \multicolumn{3}{|c|}{ Gph(up) } \\
\hline & Expt. & $L_{b}$ & $L_{a}$ & Expt. & $L_{b}$ & $L_{a}$ & Expt. & $L_{b}$ & $L_{a}$ & Expt. & $L_{b}$ & $L_{a}$ \\
\hline$A$ & 1724 & 1715 & 1710 & 1704 & 1718 & 1690 & 1587 & 1566 & 1537 & 1593 & 1579 & 1636 \\
\hline$B$ & 673 & 667 & 672 & 673 & 663 & 664 & 743 & 744 & 760 & 728 & 726 & 710 \\
\hline$C$ & 545 & 540 & 543 & 545 & 538 & 535 & 554 & 545 & 548 & 555 & 546 & 552 \\
\hline$\mu_{a}$ & 0.10 & 0.03 & 4.02 & 0.10 & 0.07 & 4.14 & $\cdots$ & 0.54 & 3.35 & 0.50 & 0.40 & 1.93 \\
\hline$\mu_{b}$ & 1.26 & 1.00 & 6.34 & 2.34 & 2.57 & 5.68 & $\cdots$ & 2.48 & 6.08 & 2.03 & 2.24 & 4.59 \\
\hline$\mu_{c}$ & 0.46 & 0.61 & 0.27 & 0.87 & 0.94 & 0.94 & $\cdots$ & 1.28 & 1.36 & 0.62 & 0.67 & 0.53 \\
\hline$\phi$ & 72 & 77 & 84 & 72 & 77 & 85 & 77 & 81 & 77 & 81 & 81 & 76 \\
\hline$\theta$ & 14 & 4 & 87 & 22 & 3 & 86 & 18 & 10 & 85 & 14 & 10 & 84 \\
\hline
\end{tabular}


TABLE V. Molecular parameters of the excited states of the anti conformers of tryptamine. The calculated transition dipole moment angles $\phi$ and $\theta$ and the permanent dipole moments are calculated by DFT/MRCI in the TDDFT optimized geometry of the respective state. Rotational constants are given in megahertz, dipole moments in debye, and angles of the transition dipole moment in degrees.

\begin{tabular}{|c|c|c|c|c|c|c|c|c|c|}
\hline & \multicolumn{3}{|c|}{ Anti(py) } & \multicolumn{3}{|c|}{ Anti(up) } & \multicolumn{3}{|c|}{ Anti(ph) } \\
\hline & Expt. & $L_{b}$ & $L_{a}$ & Expt. & $L_{b}$ & $L_{a}$ & Expt. & $L_{b}$ & $L_{a}$ \\
\hline$A$ & 1754 & 1755 & 1737 & 1749 & 1767 & 1738 & 1761 & 1768 & 1729 \\
\hline$B$ & 612 & 610 & 617 & 609 & 603 & 616 & 610 & 606 & 620 \\
\hline$C$ & 473 & 471 & 477 & 471 & 469 & 478 & 473 & 470 & 477 \\
\hline$\mu_{a}$ & $\cdots$ & 0.24 & 3.07 & 0.30 & 0.07 & 3.67 & - & 0.67 & 3.58 \\
\hline$\mu_{b}$ & $\cdots$ & 0.90 & 4.63 & 2.34 & 2.47 & 5.78 & $\cdots$ & 2.58 & 6.07 \\
\hline$\mu_{c}$ & $\cdots$ & 0.59 & 0.75 & 0.87 & 1.07 & 0.94 & $\cdots$ & 1.28 & 1.29 \\
\hline$\phi$ & 75 & 83 & 83 & 77 & 82 & 83 & 75 & 82 & 82 \\
\hline$\theta$ & 6 & 15 & 81 & 11 & 12 & 81 & 19 & 13 & 83 \\
\hline
\end{tabular}

\section{E. The tryptamine-water cluster}

\section{Electronic ground state energies and structures}

Complexation of tryptamine with water selectively stabilizes one conformer, which could be shown experimentally to be the Gpy(out) $(A)$ conformer. $^{17,24-27}$ This selectivity could be traced back to the formation of a double hydrogen bond, which connects the amino group (stronger $\mathrm{H}$ bond) and the pyrrolic $\mathrm{CH}$ group (weaker $\mathrm{H}$ bond) via the water molecule. In the following we will concentrate only on the bridged water cluster of tryptamine $A$, which is the only one that has been observed experimentally until now. Differently bound water clusters (hydrogen bonded via the pyrrolic NH group or trans-linearly bound to the amino group with water as proton acceptor $-\mathrm{NH}_{2} \cdots \mathrm{OH}_{2}$, or donor bound to the lone pair of the amino group $-\mathrm{H}_{2} \mathrm{~N} \cdots \mathrm{HOH}$ ) of other conformers of tryptamine were shown either to have considerably higher energies than the cyclic tryptamine $A$ cluster or to have completely different rotational constants than the experimentally

TABLE VI. Molecular parameters for tryptamine [Gpy(out)]-water. Ground state properties are denoted by double primes, and excited state properties by single primes. The calculated transition dipole moment angles $\phi$ and $\theta$ and the permanent dipole moments of ground and excited states are calculated by DFT/MRCI in the TDDFT optimized geometry of the respective state.

\begin{tabular}{|c|c|c|c|c|}
\hline Ground state & Expt. & $\begin{array}{c}\mathrm{MP} 2 \\
6-311 \mathrm{G}(d, p)\end{array}$ & \multicolumn{2}{|c|}{$\begin{array}{l}\text { B3LYP } \\
\text { TZVP }\end{array}$} \\
\hline$A^{\prime \prime}(\mathrm{MHz})$ & 1465.78 & 1483 & \multicolumn{2}{|c|}{1475} \\
\hline$B^{\prime \prime}(\mathrm{MHz})$ & 483.43 & 488 & \multicolumn{2}{|c|}{472} \\
\hline$C^{\prime \prime}(\mathrm{MHz})$ & 397.99 & 403 & \multicolumn{2}{|c|}{389} \\
\hline$\mu_{a}^{\prime \prime}(\mathrm{D})$ & $\ldots$ & 0.50 & \multicolumn{2}{|c|}{0.72} \\
\hline$\mu_{b}^{\prime \prime}(\mathrm{D})$ & $\ldots$ & 0.97 & \multicolumn{2}{|c|}{0.95} \\
\hline$\mu_{c}^{\prime \prime}(\mathrm{D})$ & $\cdots$ & 1.00 & \multicolumn{2}{|c|}{1.04} \\
\hline Excited state & & & TDDFT $\left(L_{b}\right)$ & TDDFT $\left(L_{a}\right)$ \\
\hline$A^{\prime}(\mathrm{MHz})$ & 1458.96 & $\ldots$ & 1462 & 1433 \\
\hline$B^{\prime}(\mathrm{MHz})$ & 478.72 & $\cdots$ & 470 & 489 \\
\hline$C^{\prime}(\mathrm{MHz})$ & 394.27 & $\ldots$ & 386 & 400 \\
\hline$\mu_{a}^{\prime}(\mathrm{D})$ & $\ldots$ & $\ldots$ & 1.54 & 6.08 \\
\hline$\mu_{b}^{\prime}(\mathrm{D})$ & $\ldots$ & $\cdots$ & 1.04 & 1.37 \\
\hline$\mu_{c}^{\prime}(\mathrm{D})$ & $\ldots$ & $\ldots$ & 1.02 & 0.94 \\
\hline$\phi\left(^{\circ}\right)$ & 65.53 & $\cdots$ & 74.9 & 85.3 \\
\hline$\theta\left(^{\circ}\right)$ & 9.40 & $\cdots$ & 18.6 & 68.9 \\
\hline
\end{tabular}

determined ones. ${ }^{19,26}$ Zwier $^{27}$ performed RIDIR spectroscopy on the cluster and deduced from the vibrational pattern a strong $-\mathrm{H}_{2} \mathrm{~N} \cdots \mathrm{HOH}$ hydrogen bond. The electronic origin of the cluster is blueshifted by only $42 \mathrm{~cm}^{-1}$, compared to the origin of the $A$ conformer. This small shift already points to a bridged cluster, since bonding to the pyrrolic NH group (water as proton acceptor, like in indole) would lead to a redshift, comparable to that in indole, while bonding to the amino group (water as proton acceptor) should result in a larger blueshift than observed. From the experimental rotational constants any $\pi$-bound structures can be excluded. The ground state geometry has been optimized at the MP2/6-311G $(d, p)$ and the B3-LYP/TZVP level of theory.

The resulting rotational constants are compared in Table VI to the experimentally determined ones. The agreement for both the MP2 and the B3-LYP calculations again is reasonably good. The structure of the tryptamine-water cluster is shown in Fig. 6. Electronic ground state energies and dipole moments of tryptamine-water have been calculated at the MP2 and the DFT/MRCI level of theory (Table VI).

The cluster stabilization energy at MP2/6-311G $(d, p)$ level including zero-point energy and BSSE correction using the counterpoise method of Boys and Bernardi ${ }^{46}$ amounts to $20 \mathrm{~kJ} / \mathrm{mol}$. This is close to the stabilization energy found for the hydrogen bonded aniline-water cluster at MP2/6-31G $(d, p)$ level including BSSE correction. ${ }^{47}$ The corresponding stabilization energies for pyrrolic $\mathrm{NH}$ bound indole-water and 1-methylindole-water complexes are 15 and $7 \mathrm{~kJ} / \mathrm{mol}$, respectively. 48

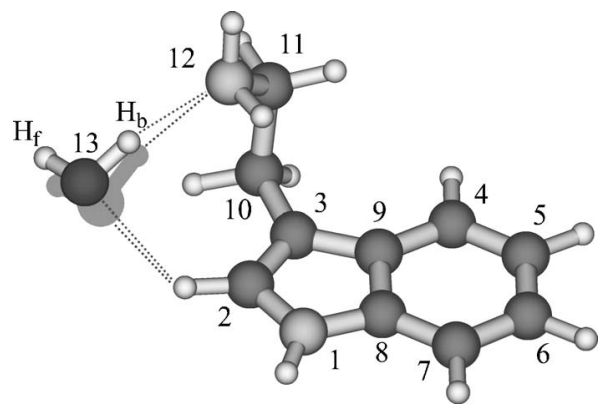

FIG. 6. Ground state structure of the tryptamine-water cluster (solid atoms) along with the principal change of the solvent orientation upon excitation to the $L_{b}$ state (shaded atoms). Solvent changes are not drawn to scale. 


\section{Vertical and adiabatic excitation spectra of the tryptamine-water cluster}

In the vertical excitation spectrum at the DFT/MRCI level, the $L_{b}$ is calculated to lie approximately $2000 \mathrm{~cm}^{-1}$ below the $L_{a}$ state (Fig. 2). Contrary to the case of the monomer, the optimization of the $L_{b}$ state poses no problem although it is not the lowest excited state at the TDDFT level. At this level of theory, the lowest excited singlet state is the $L_{a}$ and the second excited singlet state is the $L_{b}$ state. As for the monomer, the individual contributions of the two configurations $(\mathrm{HOMO}-1 \rightarrow \mathrm{LUMO}$ and $\mathrm{HOMO} \rightarrow \mathrm{LUMO}+1)$ to the electric transition to the $L_{b}$ state nearly cancel, resulting in a small oscillator strength for this state. Contrary to the monomer, the $L_{a}$ state remains the lowest electronically excited state in the adiabatic excitation spectrum at the DFT/ MRCI level, while the lowest state in the vertical excitation spectrum is the $L_{b}$ state. Nevertheless, comparing both the adiabatic excitation energies and the TDM orientation of the $L_{a}$ and $L_{b}$ states with the experimentally determined value (Table VI) it is obvious that the experimental spectrum is due to the $L_{b}$ state. The DFT/MRCI excitation energies at the respective geometries including BSSE and zero-point energy corrections for all states involved are given in Table II. The BSSE of the tryptamine-water cluster in the $L_{a}$ and in the $L_{b}$ states is calculated to be 156 and $293 \mathrm{~cm}^{-1}$, respectively, using the counterpoise method of Boys and Bernardi. The resulting blueshift of the adiabatic excitation energy of $31 \mathrm{~cm}^{-1}$ is in surprisingly good agreement with the experimentally determined value of $41 \mathrm{~cm}^{-1}$.

\section{F. Structural changes of the water cluster upon electronic excitation}

Table VI compares the experimentally determined rotational constants, the permanent dipole moments of ground and both electronically excited states, and the transition dipole moments with the calculated values. Clearly, both the geometry changes and the transition dipole moment orientation point to the $L_{b}$ as experimentally observed excited state of the tryptamine-water complex like for the monomer. The calculated ground state structures and the geometry changes upon excitation of the tryptamine-water cluster to the $L_{a}$ and the $L_{b}$ states are compiled in Table V. The changes of the chromophore geometry in the cluster are very close to those of the monomer itself. The ground state hydrogen bond length between the amino nitrogen and the hydrogen bonded hydrogen atom of the water moiety $r\left(\mathrm{~N}_{12}-\mathrm{H}_{b}\right)$ slightly decreases upon excitation to the $L_{a}$ state and remains the same for $L_{b}$. In contrast, the weak hydrogen bond between the water oxygen atom and the pyrrolic $\mathrm{C}_{2} \mathrm{H}$ group decreases strongly for both $L_{a}(-17 \mathrm{pm})$ and $L_{b}(-8 \mathrm{pm})$ states, accompanied by a strong change in the $\mathrm{C}_{11} \mathrm{~N}_{12} \mathrm{O}_{13}$ angle. Thus, the geometry change can approximately be described as a rotation of the water moiety, which leaves the $\mathrm{N}_{12}-\mathrm{H}_{b}$ distance constant, but decreases the $\mathrm{C}_{2} \mathrm{H}-\mathrm{O}_{13}$ distance. This effect can be attributed to a considerable increase of the acidity of the pyrrolic $\mathrm{CH}$ group upon electronic excitation. The large geometry change of the dihedral angle, which describes the water orientation with respect to the tryptamine moiety and the decrease in hydrogen bond length should show up in a long Franck-Condon progression of the wagging mode and possibly in the stretching mode $\sigma$ in the dispersed fluorescence spectra obtained via excitation through the electronic origin. Unfortunately, no such spectra are available yet in the literature.

\section{CONCLUSIONS}

We performed DFT/MRCI calculations for the ground state and the lowest excited singlet states of the seven experimentally observed conformers of tryptamine and the hydrogen bonded water cluster and compared the results to the experimental values. The adiabatic excitation energies of the $L_{b}$ state show a nearly perfect agreement with the experimentally determined ones, if one takes ZPE corrections for the ground and excited state energies into account. The largest absolute deviation to the experimental excitation energy amounts only to about $300 \mathrm{~cm}^{-1}$, which is less than $1 \%$ of the absolute value. Nevertheless, the sensitivity of the $L_{b}$ origin frequency on the conformation of the ethyl amino chain with respect to the chromophore is exaggerated in the MRCI calculations. While the experimental excitation energies vary within an energy range of $84 \mathrm{~cm}^{-1}$, the calculated adiabatic excitation energies differ as much as $273 \mathrm{~cm}^{-1}$ from the smallest to the largest one. The origin of the $L_{a}$ state is predicted at $900-1800 \mathrm{~cm}^{-1}$ higher energy, depending on the conformer. The smallest energy difference $L_{a}-L_{b}$ is found for the Gpy(out) conformer with $885 \mathrm{~cm}^{-1}$, the largest for anti(ph) with $1810 \mathrm{~cm}^{-1}$. In general, the variation of the $L_{a}$ excitation energy with conformation is larger than the variation of the $L_{b}$ excitation energy, which can be traced back to the larger dipole moment of the former.

The lowest electronic state in the adiabatic excitation spectrum of the monomer was found to be $L_{b}$ at the DFT/ MRCI level, reversing the ordering found by time-dependent density functional theory. The assignment of the lowest excited state as being $L_{b}$ is fully confirmed by the experimental findings. The assignments of excited states through their vertical or adiabatic excitation energies merely on the basis of nowadays very popular time-dependent DFT methods have, therefore, to be questioned in some cases. On the other hand, excited state structures can be predicted reliably using TDDFT and might serve then for subsequent calculations of the electronic properties of the excited states via density functional theory/multireference configuration interaction. The problem of correctly computing the energetic ordering of $L_{a}$ and $L_{b}$ states is well known ${ }^{49}$ and can be traced back to the fact that single reference methods such as TDDFT are not able to describe appropriately multiconfigurational states such as the $L_{b}$ state.

Complexation of tryptamine with one water molecule draws the $L_{a}$ state below the $L_{b}$ state in the adiabatic excitation spectrum. Nevertheless, the experimentally observed lowest excited singlet state is the $L_{b}$ state, although, like for the monomer, the oscillator strength of the $L_{a}$ is larger than that of the $L_{b}$ state. Since the absolute values of the adiabatic excitation energies of the conformers of the monomer and of the cluster are nearly quantitatively correct, we dare to pre- 
dict the origin of the $L_{a}$ state of the tryptamine-water complex to be redshifted by approximately $900 \mathrm{~cm}^{-1}$, compared to the hitherto exclusively observed $L_{b}$ origin. A large reorientation of the water solvent molecule has been found upon excitation to the $L_{a}$ state from the $a b$ initio calculations, which might cause a low Franck-Condon activity for this transition compared to the $L_{b}$ origin. Also, an efficient nonradiative decay channel for the $L_{a}$ state, which for several indole derivatives has been postulated to be $\mathrm{N}-\mathrm{H}$ bond fission, might prevent the experimental observation of the $L_{a}$ origin of the cluster. ${ }^{50}$

Finally, it has to be mentioned that the photophysics of tryptamine is also influenced by the existence of other electronic states, which might be energetically close. A prominent and much discussed example is the $\pi \sigma^{*}$ state, which has been postulated by Sobolewski et al. ${ }^{51}$ and by Sobolewski and Domcke ${ }^{52}$ for indole and similar chromophores and has spectroscopically been characterized by Dian et al. ${ }^{14}$ for several indole derivatives, in which a strong coupling of the hydride stretch vibrational levels to the $\pi \sigma^{*}$ dissociative continuum is observed, which results in a quenching of the $\mathrm{NH}$ stretch vibration in the electronically excited state. Theoretical and experimental studies on other electronically excited states are currently on the way.

\section{ACKNOWLEDGMENTS}

This work was supported by Deutsche Forschungsgemeinschaft through SFB 663 projects A2 and C1. The authors would like to thank the National Computer Facilities of the Netherlands Organization of Scientific Research (NWO) for a grant on the Dutch supercomputing facility SARA and the Universitätsrechenzentrum Köln for the granted computing time.

${ }^{1}$ E. M. Evleth, O. Chalvet, and P. Bamlère, J. Phys. Chem. 81, 1913 (1977).

${ }^{2}$ J. W. Hager, D. R. Demmer, and S. C. Wallace, J. Phys. Chem. 91, 5513 (1984).

${ }^{3}$ Y. D. Park, T. R. Rizzo, L. A. Peteanu, and D. H. Levy, J. Chem. Phys. 84, 6539 (1986).

${ }^{4}$ L. A. Philips and D. H. Levy, J. Chem. Phys. 89, 85 (1988).

${ }^{5}$ L. L. Connell, T. C. Corcoran, P. W. Joireman, and P. M. Felker, Chem. Phys. Lett. 166, 510 (1990).

${ }^{6}$ M. R. Eftink, L. A. Selvidge, P. R. Callis, and A. A. Rehms, J. Phys. Chem. 94, 3469 (1990).

${ }^{7}$ M. J. Tubergen and D. H. Levy, J. Phys. Chem. 95, 2175 (1991).

${ }^{8}$ T. M. Korter, D. W. Pratt, and J. Küpper, J. Phys. Chem. A 102, 7211 (1998).

${ }^{9}$ K. W. Short and P. R. Callis, J. Chem. Phys. 108, 10189 (1998).

${ }^{10}$ K. W. Short and P. R. Callis, J. Chem. Phys. 113, 5235 (2000).

${ }^{11}$ A. C. Borin and L. Serrano-Andrés, Chem. Phys. 262, 253 (2000).
${ }^{12}$ L. Serrano-Andrés and A. C. Borin, Chem. Phys. 262, 267 (2000).

${ }^{13}$ J. R. Carney and T. S. Zwier, J. Phys. Chem. A 104, 8677 (2000).

${ }^{14}$ B. C. Dian, A. Longarte, and T. S. Zwier, J. Chem. Phys. 118, 2696 (2003).

${ }^{15}$ K. R. F. Somers and A. Ceulemans, J. Phys. Chem. A 108, 7577 (2004).

${ }^{16}$ W. Caminati, Phys. Chem. Chem. Phys. 6, 2806 (2004).

${ }^{17}$ B. C. Dian, J. Clarkson, and T. S. Zwier, Science 303, 1169 (2004).

${ }^{18}$ T. Nguyen, T. Korter, and D. Pratt, Mol. Phys. 103, 1603 (2005).

${ }^{19}$ M. Schmitt, M. Böhm, C. Ratzer, C. Vu, I. Kalkman, and W. L. Meerts, J. Am. Chem. Soc. 127, 10356 (2005).

${ }^{20}$ J. R. Clarkson, B. C. Dian, L. Moriggi, A. DeFusco, V. McCarthy, K. D. Jordan, and T. S. Zwier, J. Chem. Phys. 122, 214211 (2005).

${ }^{21}$ T. Nguyen and D. Pratt, J. Chem. Phys. 124, 054317 (2006).

${ }^{22}$ M. Böhm and M. Schmitt (unpublished).

${ }^{23}$ L. A. Peteanu and D. H. Levy, J. Chem. Phys. 92, 6554 (1988).

${ }^{24}$ J. Sipior and M. Sulkes, J. Chem. Phys. 88, 6146 (1988).

${ }^{25}$ P. M. Felker, J. Phys. Chem. 96, 7844 (1992).

${ }^{26}$ L. L. Connell, T. C. Corcoran, P. W. Joireman, and P. M. Felker, J. Phys. Chem. 94, 1229 (1990).

${ }^{27}$ T. S. Zwier, J. Phys. Chem. A 105, 8827 (2001).

${ }^{28}$ J. R. Carney, B. C. Dian, G. M. Florio, and T. S. Zwier, J. Am. Chem. Soc. 123, 5596 (2001).

${ }^{29}$ R. Ahlrichs, M. Bär, M. Häser, H. Horn, and C. Kölmel, Chem. Phys. Lett. 162, 165 (1989).

${ }^{30}$ A. Schäfer, C. Huber, and R. Ahlrichs, J. Chem. Phys. 100, 5829 (1994).

${ }^{31}$ P. J. Stephens, F. J. Devlin, C. F. Chabalowski, and M. J. Frisch, J. Phys. Chem. 98, 11623 (1994).

${ }^{32}$ C. Lee, W. Yang, and R. Parr, Phys. Rev. B 37, 785 (1988).

${ }^{33}$ F. Furche and R. Ahlrichs, J. Chem. Phys. 117, 7433 (2003).

${ }^{34}$ R. Ahlrichs, M. Bär, H.-P. Baron, R. Bauernschmitt, S. Böcker, P. Deglmann, M. Ehrig, K. Eichkorn, S. Elliott, and F. Furche, tURBOMOLE, Version 5.6, Universität Karlsruhe, Germany, 2002.

${ }^{35}$ M. J. Frisch, G. W. Trucks, H. B. Schlegel et al., GAUSSIAN 03, Revision A.1, Gaussian, Inc., Pittsburgh, PA, 2003.

${ }^{36}$ O. Treutler and R. Ahlrichs, J. Chem. Phys. 102, 346 (1995).

${ }^{37}$ F. Weigend and M. Häser, Theor. Chem. Acc. 97, 331 (1997).

${ }^{38}$ F. Weigend, M. Häser, H. Patzelt, and R. Ahlrichs, Chem. Phys. Lett. 294, 143 (1998).

${ }^{39}$ J. T. H. Dunning, J. Chem. Phys. 90, 1007 (1989).

${ }^{40}$ J. W. Ochterski, G. A. Petersson, and J. J. A. Montgomery, J. Chem. Phys. 104, 2598 (1996).

${ }^{41}$ J. J. A. Montgomery, M. J. Frisch, and J. W. Ochterski, J. Chem. Phys. 112, 6532 (1996).

${ }^{42}$ S. Grimme and M. Waletzke, J. Chem. Phys. 111, 5645 (1999).

${ }^{43}$ A. D. Becke, J. Chem. Phys. 98, 1372 (1993).

${ }^{44}$ See EPAPS Document No. E-JCPSA6-125-305638 for the experimental and calculated ground state rotational constants and dipole moments of the seven tryptamine conformers. This document can be reached via a direct link in the online article's HTML reference section or via the EPAPS homepage (http://www.aip.org/pubservs/epaps.html).

${ }^{45}$ L. Serrano-Andrés and B. O. Roos, J. Am. Chem. Soc. 118, 185 (1996).

${ }^{46}$ S. F. Boys and F. Bernardi, Mol. Phys. 19, 553 (1970).

${ }^{47}$ U. Spoerel and W. Stahl, J. Mol. Struct. 190, 278 (1998).

${ }^{48}$ J. R. Carney and T. S. Zwier, J. Phys. Chem. A 103, 9943 (1999).

${ }^{49}$ M. Parac and S. Grimme, J. Phys. Chem. A 106, 6844 (2002).

${ }^{50}$ N. Glasser and H. Lami, J. Chem. Phys. 74, 6526 (1981).

${ }^{51}$ A. L. Sobolewski, W. Domcke, C. Dedonder-Lardeux, and C. Jouvet, Phys. Chem. Chem. Phys. 4, 1093 (2002).

${ }^{52}$ A. L. Sobolewski and W. Domcke, Chem. Phys. Lett. 315, 293 (1999). 